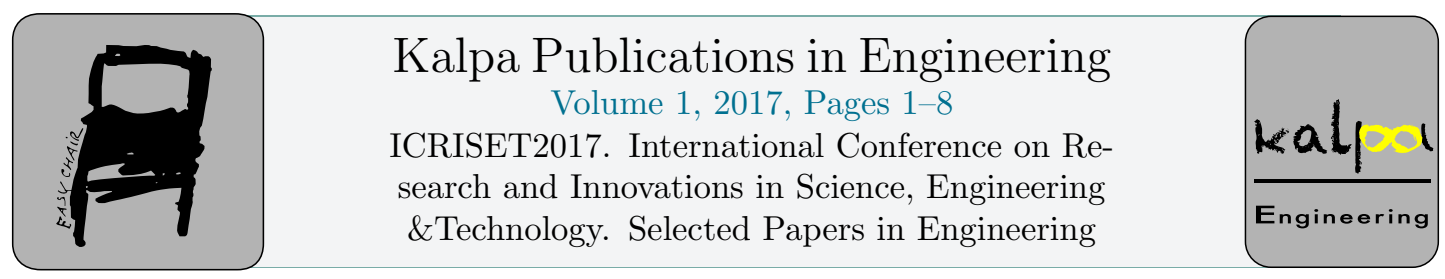

\title{
ALPR System Under Varying Illumination and Motion Conditions
}

\author{
H. N. Patel ${ }^{1}$ and T. Panchal ${ }^{2}$ \\ ${ }^{1}$ Professor, Electronics and Communication Engg. Department, A.D. Patel Institute of \\ Technology,New V.V.Nagar, Anand, Gujarat, India \\ ${ }^{2}$ PG Student, Electronics and Communication Engg. Department, A.D. Patel Institute of \\ Technology,New V.V.Nagar, Anand, Gujarat, India \\ ec.hetal.patel@adit.ac.in, panchaltejendra@gmail.com
}

\begin{abstract}
As the significance of open transportation framework expands a programmed license plate recognition (ALPR) has ended up being a critical examination issue. It is getting to be hard to monitor every vehicle for motivation behind law implementation and movement administration. ALPR prepared in numerous wise observation frameworks like, traffic management, security administration, programmed toll gathering framework and so forth. A number of methods have been proposed for license plate detection, each having its own advantages and disadvantages. The basic step in ALPR is accurate localization of number plate, segmentation, recognition. Algorithm for Automatic License Plate Recognition system have been developed for college premises successfully for image and video data. GMM based algorithm was carried out for extraction of license plates from video data by integrating the different controlling parameters. License plates are detected from a video frame effectively by using Harris corner detector. Segmentation results achieved was good by utilizing integrated approach of controlling parameters. Artificial neural network (ANN) had used for the recognition purpose and accuracy achieved was high, also data logging had been implemented successfully for future purpose. Keywords-ALPR, LP, SWA, CCA, AR, PC, ANN
\end{abstract}

\section{Introduction}

The raising augmentation of contemporary urban and National Street monitoring all through the latest three decades emerged the need of capable watching and organization of street traffic movement. Automatic License Plate Recognition (ALPR) is an imaging innovation connected to distinguish plates for their vehicles. In actuality, frameworks that depend on video are anything but difficult to introduce, utilize the current base of activity reconnaissance. Moreover, they can be 
effortlessly overhauled and they offer the adaptability to update the plan and its usefulness by just changing the framework calculations. Those frameworks permit estimation of the vehicles speed, considering the quantity of vehicles, grouping of vehicles, and the recognition of movement episodes, (for example, chance occasions or overwhelming clog). Particular lighting conditions can impact an extensive measure in the general segmentation rate. There is an expansive assortment of courses of action taking into account video and picture preparing utilizing distinctive philosophies to discover vehicles and distinguish. Issues, for example, shadow, night photography, and recognition rate are additionally tended to, when accessible. Detail study by authors in (C.-N. Anagnostopoulos, 2008)(C. Patel, 2013) had done for ALPR frameworks.

A flawless License Plate Localization strategy typically upgrades the accuracy of the entire framework and prompts a quick affirmation process. For progressing application, the speed and precision of the systems ought to be gained ground. This errand is testing as a result of a couple of constituents that can incite low quality pictures. Some of the affecting factors are, brightening conditions, rotation, broken character, non-LP local character, Blurring effect etcetera.

This paper exhibits a framework that can oversee particular light conditions, (for example, splendor, nightshade, shadow, etcetera.) furthermore with distortion conditions. Thus a few caught pictures of various sorts of vehicles in assorted ending conditions and check impacts have been managed to show the authenticity of the proposed strategy. This paper is organized as follows. In section 2, related work is talked about in a general and in part 3 the proposed strategy is depicted with stream chart. The observational results are displayed in section 4 . At last, the paper is closed in part 5.

\subsection{Literature review}

Generally, For the most part, ALPR recognition framework works in four essential steps. 1) Frame extraction 2) Plate detection 3) character segmentation and 4) character recognition. The paper presented LP detection and segmentation procedure and move over a brief history of the right now accessible techniques used as a piece of ALPR framework. The particular systems are accessible for LP detection, for example, Histogram based(Z. Baohua, 2010), Otsu based thresholding technique, Wavelet based detection(C. N. Paunwala and S. Patnaik, 2010), Projective investigation (Patnaik), Fuzzy rationale (C. N. Paunwala, 2012), Rectangle/window seek (N. F. Gazc'on, 2012), Sliding window-Haar (Y.-r. Wang, 2011)etcetera. Where regarding the segmentation, Horizontal/vertical projection (Abderaoufi, 2014), (J. Jagannathan, 2013), Blob examination (Y. Yoon K.-d. B., 2011), (Y. Yoon K.-d. B., 2012), Watershed calculation (H.-m. Interface, 2010), Contour, OCS (I. Giannoukos, 2010) (Operator Context Scanning), Thinning-Thresholding, Integrated approach (T. Panchal, 2016), CCA (N. Vishwanath, 2012), (M. K. Sarker, 2014) and so forth. For Recognition of extracted characters, Template matching technique (Tanmoy, 2015), Intelligent Template Matching (ITM) (S. Goel, 2013), statistical and structural recognition methods (X. Pan, 2005), Probabilistic Neural Network (PNN) (A. E. Ghahnavieh, 2014) is used so far.

\section{Proposed Method}

In this section, we are discussing the proposed algorithm when all is said in done, and after that we are portraying the two noteworthy parts of our system one by one, in the following parts. Figure 1 shows a review of the ALPR system. Initially a video/picture was taken by a camera and it was considered as an input frames/image. After extracting the frame from video, Detection part is carried out and then the detected plate region is feed forward for the Segmentation. Here, an integrated approach is taken into consideration for getting good result in segmentation. Once the LP characters are segmented, recognition using ANN method had been carried out. 


\subsection{Frame Extraction}

Gaussian Mixture Model (GMM) algorithm had been used to estimate the foreground object from the background within video. All the parameters for detecting any moving objects had been set based upon the observations. Once the object is detected, decision needs to be taken whether or not any vehicle present in that particular frame. For that, Area and horizontal length had been consider as controlling parameter. Area is used to determine that weather big object (such as, car, different vehicles) is present in the extracted frames or not. Horizontal length also been used as a parameter for improvising the reliability to frame extraction technique. It won't allow the frame to be processed for detection stage in case where no vehicle is present and some other objects are present. Figure 2 shows the result of extracted frame from video to be feed forward in detection stage.

\subsubsection{LP Detction}

Once the frame extracted, detection of a license plate with the frame needs to be detected. Harris corner algorithm had been utilized to detect the corner points in the frame. Usually characters have more number of points than other region in an image. Scanning Window Analysis (SWA) technique is used to find the corner points and make cluster which might the probable region for LP in a frame. Different parameters like, area, width, height, aspect ratio had been set to find LP from the all probable regions after SWA (T. Panchal, 2016). Figure 3 shows the results obtained after applying detection strategy on the extracted frame. The input image was filtered with the mask size of $[u, v]$ and weights $W u . v$ for generation of Ex,y.(eq. 1). The result of filtering can be represented as the horizontal and vertical line crossing detection.

$$
E_{x, y}=\sum w_{u, v}\left[I_{x+u, y+v}-I_{u, v}\right]^{2}=A x^{2}+2 C x y+B y^{2}
$$

$$
\text { Where, } A=X^{2} \otimes w, B=Y^{2} \otimes w, C=(X Y) \otimes w
$$

In eq.1, $w$ represents the windowing function used to filter the image. This window can be used as gaussian or rectangle. I represent the image pixel at location $(x, y)$ with the filtering to be done by window size of $(u, v) . E_{x, y}$ gives the sum of square difference within the window size.

By using all the above equations, symmetric matrix can be defined as,

$M=\left[\begin{array}{ll}A & C \\ C & B\end{array}\right]$

Then, the feature point of Harris can be extracted as the maximum of the local area,

$R=\operatorname{Det}(M)-k \operatorname{Tr}^{2}(M)$

In the above equations, $\operatorname{Tr}$ stands for the trace of matrix whereas the Det for determinant of matrix $M$. Equation 3 is representing the corner value $R . R$ is positive for corners, negative for edges, and small for flat regions.

\subsubsection{Character Segmentation}

Detected LP is then resized using bi-cubic interpolation technique to achieve better result in segmentation of a characters/numbers. Blurring effect is not desirable while applying the thresholding as it can make thresholding operation not effective to generate good result.

So, motion effect should be expelled before applying for the segmentation part. Lucy - Richardson algorithm (J. L. Wu, 2012) had been used to remove such motion effects by assuming that motion noise having Gaussian distribution. After that, adaptive thresholding had used to binarize a LP. Adaptive thresholding had been used to achieve better result compare to Otsu (Gui-mei Zhang, 2011) method. 


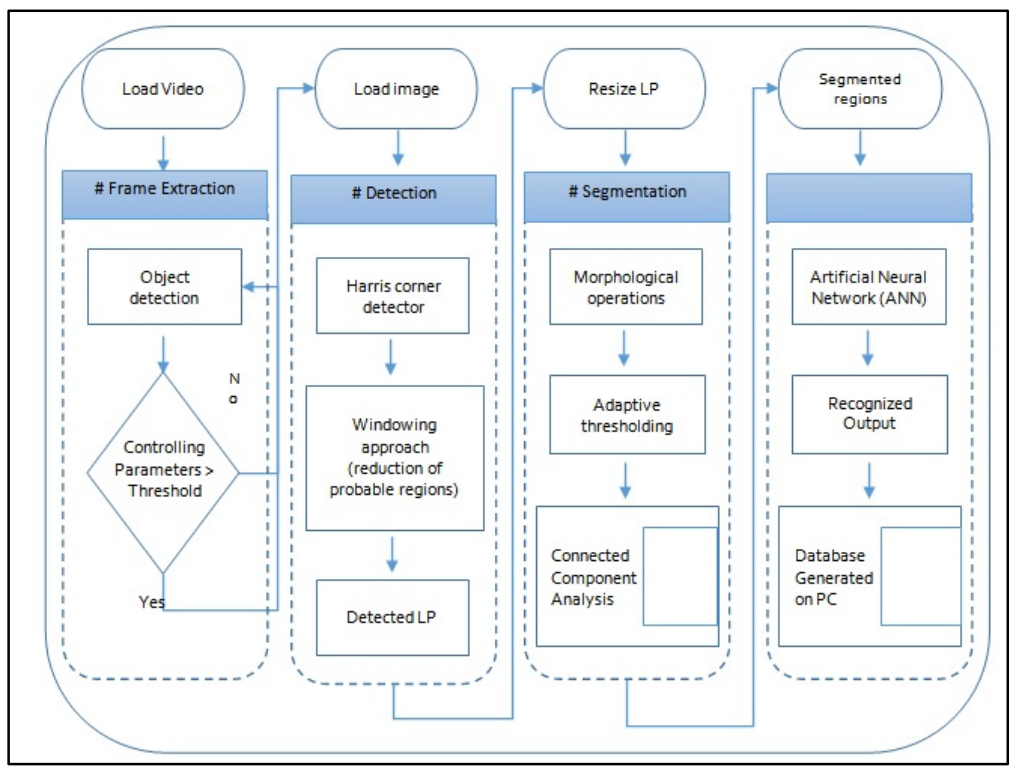

Figure 1 Proposed Algorithm

Here the window of $11 \times 11$ had been used for adaptive thresholding. Morphological operations then applied to remove noise. Connected Component Analysis (CCA) had been utilized to segment the characters from the LP. Parameters such as, Pixel Count (PC), Height, Aspect ratio had taken in count to successfully segment out the characters within LP (T. Panchal, 2016). Figure 4 shows the result obtained after applying all the controlling parameters. Red highlighted part in Figure 4(b) will not be extracted because that regions will not satisfy the aspect ratio conditions.

Same for the yellow and blue highlighted regions will not be segmented because they will not fit in area and height parameters respectively. This all parameters had set as a soft threshold which is very advantageous as it will change itself in algorithm to achieve good result in segmenting the characters.

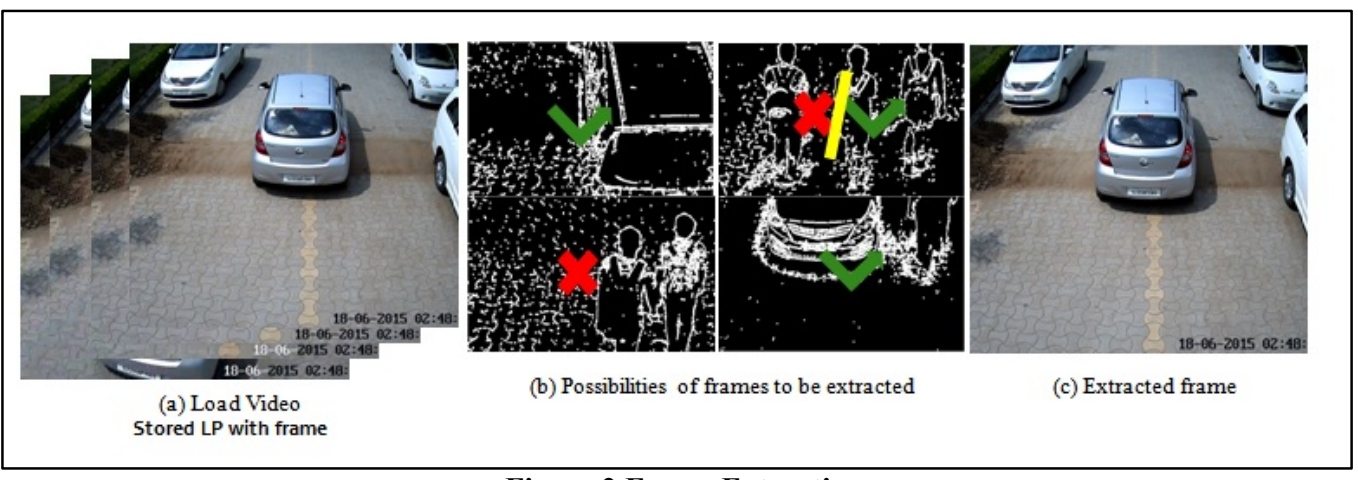

Figure 2 Frame Extraction 


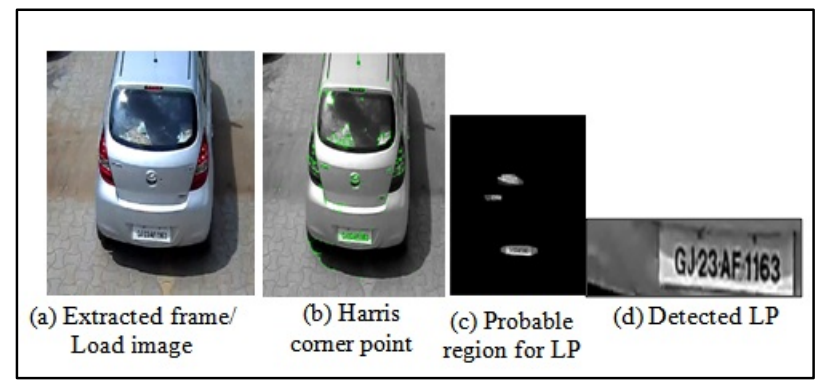

Figure 3 License Plate Detection

\subsubsection{Character Recognition}

Artificial Neural Network (ANN) had been used to recognize the segmented characters. LevenbergMarquardt algorithm (LMA) had been used as it gives the best match for weighting the neurons during the training period. Input data having total 85 node whereas hidden layer consist of 120 layers with logsig function for distribution. Output layer have total 23 classified classes by using tansig used during training. Once the network had trained with large dataset of around 300 characters, it's been tasted and matched up-to to the higher accuracy level. The result of recognition can be seen in the Figure 5. LP from the extracted frame had been successfully recognized.

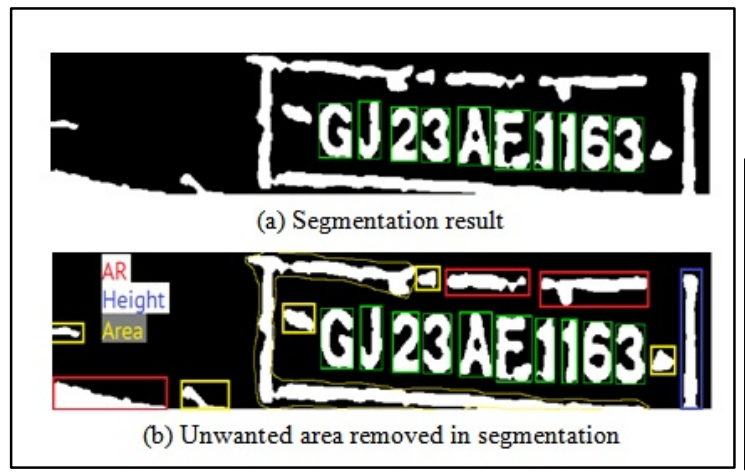

Figure 4 Segmentation Result

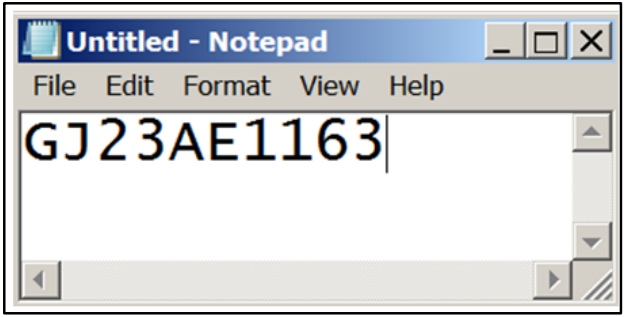

Figure 5 Recognition Result

\section{Simulation results}

In this section, the experimental results of the proposed system for localization and segmentation and recognitions are presented. The system said to be robust if it's able to extract the license plate from the image and segmented the characters correctly with good recognition accuracy. The results shown in Figure 4 are the one obtained by implementing the proposed algorithm in licensed software MATLAB $^{\circledR}$. Different illumination conditions are taken care while doing the simulation. For the blurred images, segmentation is typical task to attain. For highly blurred images, better adaptive thresholding is needed. Recognition accuracy can also be increased by including different types of characters in training dataset to make network robust. It can be seen from the Figure 6 that algorithms give good result of recognition under different illumination and motion conditions.

Also the data-log of vehicle with the extracted LP had stored at local storage to be used for the future purpose. Figure 7 shows the data log generated automatically from the video data. 


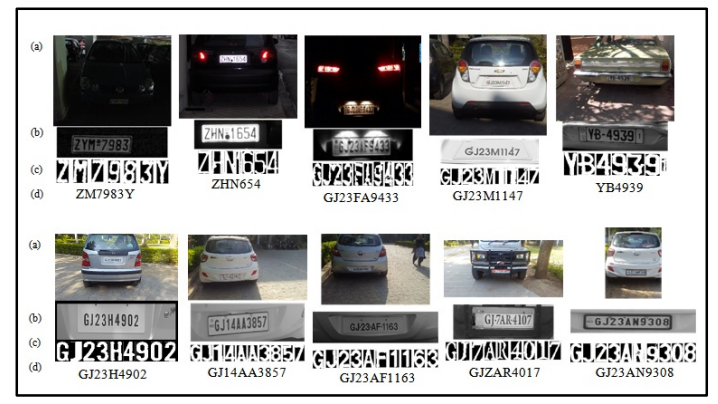

Figure 6 Experiment Results

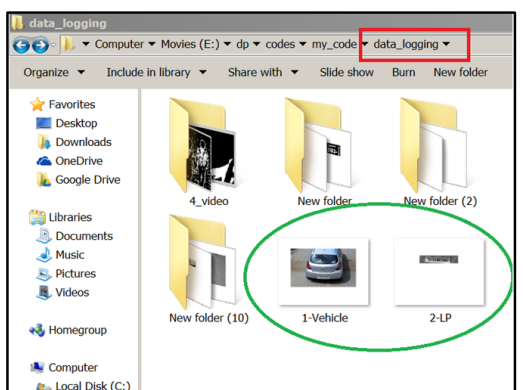

Figure 7 Data-Log stored at Local storage

Results obtained for video data are very promising as it gives good recognition results and 100\% detection results. It can be seen from the Table. I that this algorithm gives very good recognition rate for images in diverse conditions. Recognition rate for the video data is not much higher but it's because of low resolution quality video data gives blurred images/frames and hence the recognition results not up-to the mark. The proposed algorithm will work very well where the quality of video is high and resolution is more.

Table 1 Experimental results

\begin{tabular}{|l|c|c|c|c|}
\hline \multirow{2}{*}{ Input data } & \multirow{2}{*}{$\begin{array}{l}\text { Data } \\
\text { base }\end{array}$} & \multicolumn{3}{|c|}{ Accuracy (\%) } \\
\cline { 3 - 5 } & & Detection & Segmentation & Recognition \\
\hline Good images & 35 & 97.14 & 94.28 & 91.42 \\
\hline Difficult images & 30 & 93.33 & 90.00 & 86.66 \\
\hline Video & 8 & 100 & 80 & 70 \\
\hline
\end{tabular}

\subsection{Comparison with existing Technique}

The proposed technique is checked with the existing technique proposed by the author in (Bei CHEN, 2008). As the position of the vehicle and speed of the vehicle is dynamic in the input video used, the technique proposed by author (Bei CHEN, 2008) is not able to detect only the license plate, but additional components like black iron grill, extra portions around the License plate and backside bumper. The comparison between the proposed technique and the technique suggested by (Bei CHEN, 2008) is shown in Figure 8.

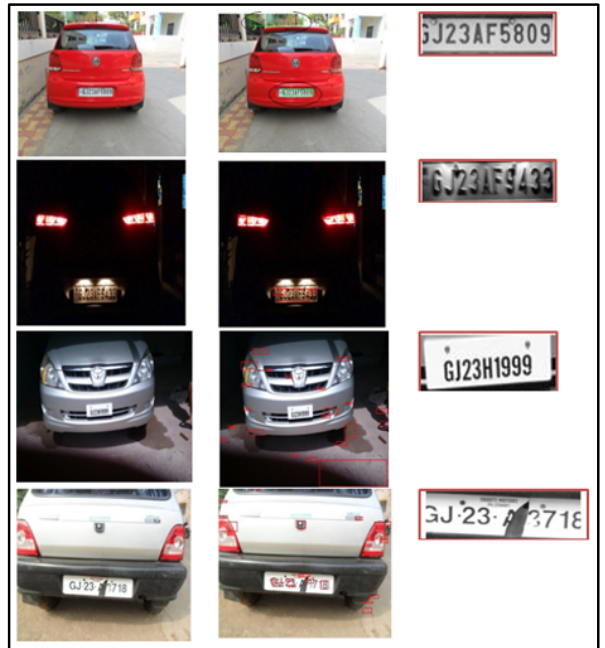

Figure 8 (a): Input Image, (b) License plate detection using [25], (c) Result of proposed technique 


\section{Conclusion}

An algorithm for Automatic License Plate Recognition system has been developed successfully for image and video data. GMM based algorithm was carried out for extraction of license plates from video data by integrating the different controlling parameters. Vehicles are detected from a video effectively and Data-Log of license plates has been generated successfully by taking care of most of constraints at college premises. Compelling results are gotten for images and video recordings having diverse difficulties for example, broken number plate, number plate in night mode, distinctive illumination conditions and motion variations so forth.

\section{Acknowledgment}

Authors are grateful to GUJCOST for providing financial assistance through minor research project 2013-14 grant. Authors also grateful to Principal and Head, Electronics and Engineering division of the A. D. Patel Institute of Technology for continuous motivation, assistance and encouragement. Authors are thankful to Dipanjali, PG Student for helping in drafting the research paper.

\section{References}

A. E. Ghahnavieh, A. A.-S. (2014). Enhancing the license plates character recognition methods by means of SVM. IEEE 22nd Iranian Conference on Electrical Engineering (ICEE), (pp. 220225).

Abderaoufi, Z. (2014). License plate character segmentation based on horizontal projection and connected component analysis. World Symposium on Computer Applications \& Research (WSCAR), 2-6.

Bei CHEN, W. C. (2008). An Efficient Algorithm on Vehicle License Plate Location. IEEE International Conference on Automation and Logistics Qingdao. China.

C. N. Paunwala and S. Patnaik. (2010). An Improved License Plate Extraction Technique Based on Gradient and Prolonged Haar Wavelet Analysis. International Conference and Workshop on Emerging Trends in Technology, (pp. 618-622).

C. N. Paunwala, S. P. (2012). An adaptive integrated rule-based algorithm for license plate localization. Opto-Electronics Review (pp. 323-334). Springer.

C. Patel, D. S. (2013). Automatic Number Plate Recognition System (ANPR): A Survey. International Journal of Computer Applications, 21-33.

C.-H. Chen, T.-Y. C.-T.-T.-C. (2013). License Plate Recognition for Moving Vehicles Using a Moving Camera. IEEE 9th International Conference on Intelligent Information Hiding and Multimedia Signal Processing, (pp. 497-500).

C.-N. Anagnostopoulos, I. A. (2008). License Plate Recognition From Still Images and Video Sequences: A Survey. IEEE Transactions on Intelligent Transportation Systems, 377-391.

Gui-mei Zhang, S.-p. C.-n. (2011). Otsu image segmentation algorithm based on morphology and wavelet transformation. 3rd International Conference on Computer Research and Development (ICCRD), (pp. 279-283). Shanghai.

H.-m. Interface, L. C.-y.-h. (2010). Vehicle License Plate Character Segmentation Method Based on Watershed. IEEE International Conference on Machine Vision and Human-machine Interface Vehicle, (pp. 447-452).

I. Giannoukos, C.-n. A. (2010). Operator context scanning to support high segmentation rates for real time license plate recognition. Pattern Recognition,Elsevier, , 3866-3878. 
J. Jagannathan, A. S. (2013). License Plate Character Segmentation Using Horizontal And Vertical Projection with Dynamic Thresholding. IEEE International Conference on Emerging Trends in Computing, Communication and Nanotechnology, (pp. 700-705).

J. L. Wu, C. F. (2012). An improved Richardson-Lucy algorithm for single image deblurring using local extrema filtering. International Symposium on Intelligent Signal Processing and Communications Systems (ISPACS), 27-32.

M. K. Sarker, M. K. (2014). A Novel License Plate Character Segmentation Method for Different Types of Vehicle License Plates. IEEE International Conference on Information and Communication Technology Convergence (ICTC), (pp. 84-88).

N. F. Gazc'on, C. I. (2012). Automatic vehicle identification for Argentinean license plates using intelligent template matching. Pattern Recognition Letters, Elsevier, 1066-1074.

N. Vishwanath, M. R. (2012). Connected Component Analysis for Indian License Plate Infra-Red and Color Image Character Segmentation. IEEE International Conference on Computational Intelligence \& Computing Research (ICCIC), (pp. 1-4).

Patnaik, C. N. (n.d.). A Novel Multiple License Plate Extraction Technique for Complex Background in Indian Traffic Conditions. International Journal of Image Processing (IJIP), 106-118.

S. Goel, S. D. (2013). Vehicle Registration Plate Recognition System Using Template Matching. IEEE International Conference on Signal Processing and Communication (ICSC), (pp. 315$318)$.

T. Panchal, H. P. (2016). License Plate Detection using Harris Corner and Character Segmentation by Integrated approach from an Image. Procedia Comput. Sci., Elsevier, 419-425.

Tanmoy, R. H. ( 2015). An Automated Vehicle License Plate Recognition System. IEEE Computer Society.

X. Pan, X. Y. (2005). A hybrid method for robust car plate character recognition. Engineering Applications of Artificial Intelligence, Elsevier, 963-972.

Y. Yoon, K.-d. B. (2011). Blob Extraction based Character Segmentation Method for Automatic License Plate Recognition System. IEEE International Conference on Systems, Man, and Cybernetics (SMC), (pp. 2192-2196).

Y. Yoon, K.-d. B. (2012). Blob Detection and Filtering for Character Segmentation of License Plates. IEEE 14th International Workshop on Multimedia Signal Processing (MMSP), 349-353.

Y.-r. Wang, W.-h. L.-j. (2011). A sliding window technique for efficient license plate localization based on discrete wavelet transform. Expert Systems With Applications, Elsevier, 31423146.

Z. Baohua, Y. D. (2010). License Plate Location Algorithm Based on Histogram Equalization. IEEE International Conference on Computer Design and Applications (ICCDA), (pp. 7-9). 